\title{
Managing Energy Consumption of Wireless Sensors Networks in Multiple Greenhouses
}

\author{
Haythem Alhumud, Mohammed Zohdy \\ Engineering and Computer Science Department, Oakland University, Rochester Hills, USA \\ Email: halhumud@Oakland.edu
}

How to cite this paper: Alhumud, $\mathrm{H}$. and Zohdy, M. (2018) Managing Energy Consumption of Wireless Sensors Networks in Multiple Greenhouses. Wireless Engineering and Technology, 9, 11-19. https://doi.org/10.4236/wet.2018.92002

Received: December 30, 2017

Accepted: April 5, 2018

Published: April 9, 2018

Copyright $\odot 2018$ by authors and Scientific Research Publishing Inc. This work is licensed under the Creative Commons Attribution International License (CC BY 4.0).

http://creativecommons.org/licenses/by/4.0/

(c) (i) Open Access

\begin{abstract}
An extensive area implementation of fully observed greenhouses motivates on research, especially in remote greenhouses. However, implementation of wireless sensor networks (WSNs) is still needed for investigation. Cognitive radio sensor networks (CRSNs) took advantage of using the cognitive radio (CR) concept to which allowed wireless sensor networks to dynamically access into white space channels which is unused channels. In this paper, we adopted the Generalized Implicit-OR as CRSN sensing protocol to reduce the energy consumption and increase the network lifetime in multiple numbers of greenhouses. Our results showed that enhanced energy consumption and improved network lifetime compared to ordinary WSN.
\end{abstract}

\section{Keywords}

Cognitive Radio, Wireless Sensor Networks, Energy-Efficient, Cooperative Sensing, Greenhouse

\section{Introduction}

The world is moving forward to fully observed agriculture, especially in greenhouses [1]. This fully observed greenhouse attracts the investors to implement in an extensive area. However, large-scale implementation of wireless sensor networks (WSNs) in greenhouses increases the spectrum scarcity problem, the scalability problem, and the energy consumption problem [2]. Cognitive radio wireless sensor networks (CRSNs) are one of the candidate areas where CR techniques can be used. Research in this area is still in its infancy, but it is progressing rapidly [3]. Adding the CR feature solves the spectrum scarcity problem and benefits from the opportunistic channel usage for bursty traffic. However, the CR concept also brings additional challenges and leads to higher energy consumption, while WSNs use energy limited devices as it is battery-powered 
[4]. The CRSN node differs from WSN node from the node, network and protocol architectures points of view. These differences made WSN techniques not applicable to CRSNs and need to adapt to the particular CRSN environment. More specifically, channel sensing protocols in CRSNs need to be energy-efficient, scalable, lightweight and have minimum channel switching rate. Otherwise, the CRSN will have smaller lifetime, higher cost, and limited number of nodes. Motivated with improving the CRSN energy efficiency, the generalized implicit-OR rule (GI-OR) protocol in CRSN can be implemented in greenhouse applications [5]. This approach solves greenhouse sensors problem in terms of efficient use of both energy and spectrum with the scalability capability. In this paper, we will adopt the GI-OR protocol in greenhouse applications for different sensor types (i.e. temperature, humidity, $\mathrm{CO}_{2}$, Soil... etc.). We compare the results between non-CR-WSN, CR WSN and the GI-OR CR WSN [6] [7]. The remainder of the paper is organized as follows. In Section 2, we present the related work. The system model and motivation are proposed in Section 3, Sections 4 and 5 are the results and discussion then the conclusions, respectively.

\section{Related Work}

In dense greenhouse sensors allocation, related works separate the greenhouse nodes, which operates in the same frequency band, from its neighboring greenhouses because of the spectrum scarcity problem [8] [9]. Figure 1 shows the WSN sensors distribution within the practically chosen greenhouses dense allocation. As shown in Figure 1, the WSN monitors only half of the greenhouses by the same operating frequency band, which leads to non-optimum occupancy. On the other hand, CR-WSN is able to reuse the same spectrum by its cognition techniques. The CR-WSN may not separate the greenhouse nodes, which operates in the same frequency band, from its neighboring greenhouses. Figure 2 shows the CR-WSN sensors distribution within the practically chosen greenhouses dense allocation. As shown in Figure 2, the CR-WSN monitors all greenhouses by the same operating frequency band.

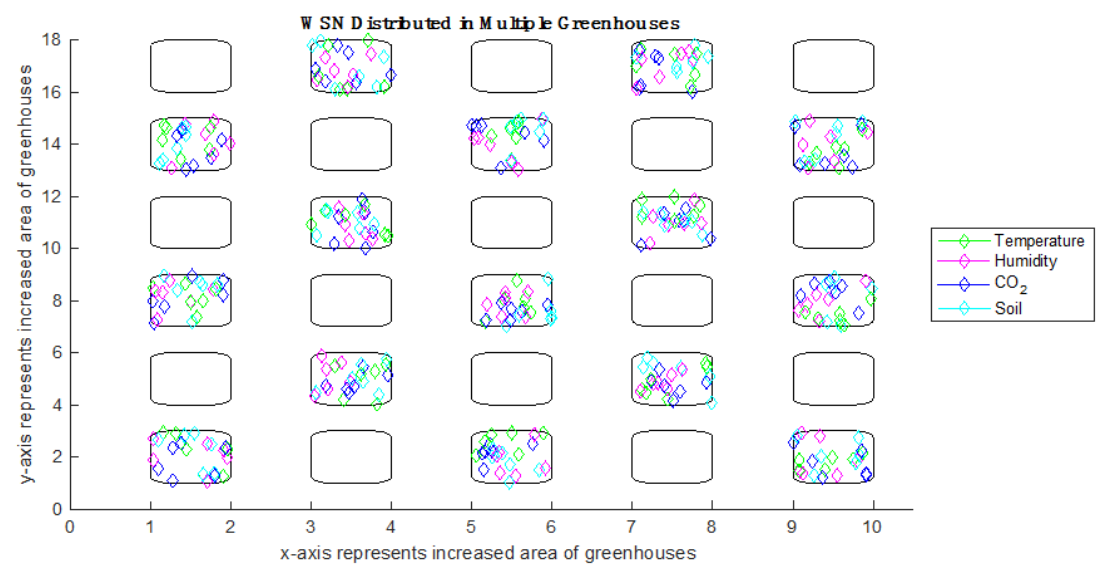

Figure 1. WSN sensors distribution of 30 greenhouses (Half occupancy due to spectrum scarcity). 


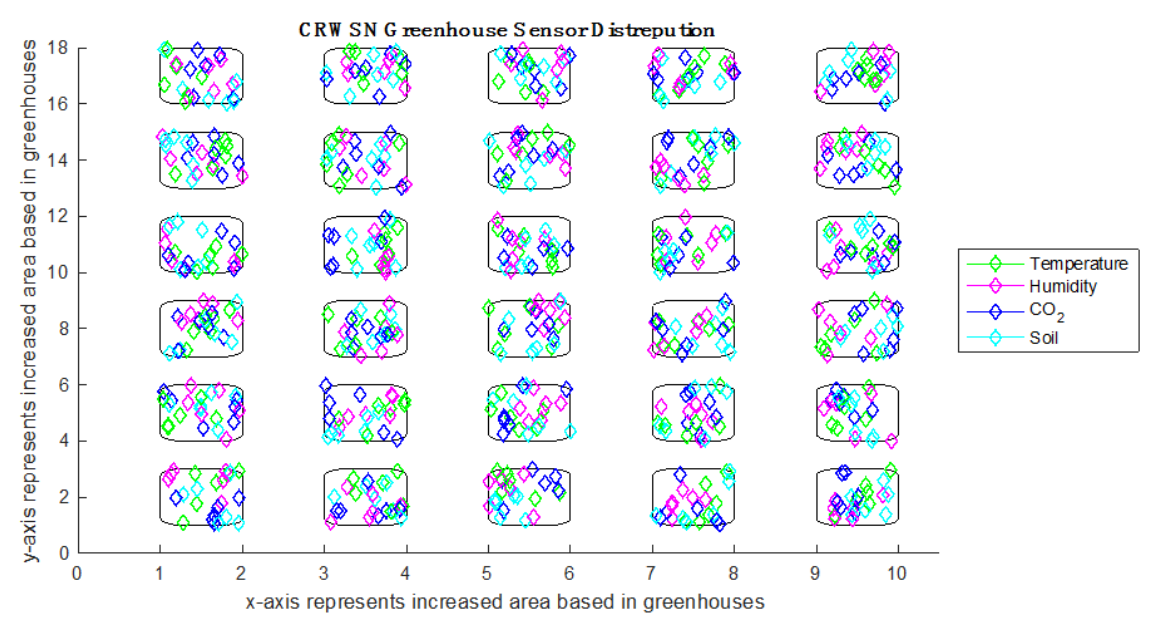

Figure 2. CR WSN sensors distribution of 30 greenhouses (full occupancy because it solves the spectrum scarcity problem).

\section{System Model \& Motivation}

\section{A. System Model}

We consider a distributed wireless CRSN geographically collocated with $\mathrm{N}$ greenhouse. Each sensor type operates in a separate channel. Each channel alternates between ON and OFF states. The OFF time represents the absence of activity, and can be exploited by the CRSN nodes. The activity factor FP is the percentage of time the channel is in the $\mathrm{ON}$ state relative to the total time, i.e. FP $=\mathrm{TON} /(\mathrm{TON}+\mathrm{TOFF}) . \mathrm{A}$ CRSN node operates in any of the $\mathrm{N}$ greenhouse. The CRSN is composed of M nodes, which are uniformly distributed within the field. We assume high density of the CRSN nodes as the case in typical wireless sensor network applications. All CRSN nodes are considered to be static. Each SU is battery powered, resource constrained and equipped with a single half-duplex transceiver. Deployed sensor nodes are homogenous in terms of hardware and initial battery power. Over time, the sensor nodes may be left out with non-uniform level of energy.

\section{B. Application Characteristic.}

WSN can only manage small scale networks. In contrast, CR sensing techniques are designed for large scale networks in which energy consumption is not of a paramount importance. Applying such techniques in CRSNs results in poor performance in terms of energy consumption, delay, required number of channels, and the required computational power [4]. In addition to the scalability problems [8], since a CRSN has much more number of nodes than WSN network. In what follows, we briefly demonstrate each of such problems.

1) Frequency Reuse: The $C R$ techniques allow wireless sensor networks to dynamically access the available channels and benefits from the opportunistic channel usage for bursty traffic.

2) High Energy Consumption: Reducing energy not only makes CRSN green but also increases the network efficiency, lifetime, reduces the required resources, and consequently, reduces the cost of the node [10] [11]. 
3) Long Delay: Large scale networks have high packet delay in multi hop buffers which leads to buffers over folding and packets lost. Moreover, reducing the decision validity interval reduces the throughput of the network, and hence, is undesirable. Consequently, the delay should be reduced to better track the activities of the greenhouse.

4) Computational Complexity: The high computational resource challenge is not suitable for WSN. Moreover, the algorithms with computational resources that scale up with the number of cooperating nodes are not feasible. This increases the complexity of the network making it a special network with higher cost. In contrast, a simpler process will allow any ordinary network (with modest nodes computational capabilities) handles the sensing task using its resources.

5) Scalability Problem: Existing WSN techniques are not scalable due to two facts. First, sensing a large amount of data requires too long transmission period. Second, a large number of nodes requires long contention period in order to convey all their information to the sink. The sink waits until all nodes senses then send their information, which will be no longer valid data after this too long delay.

This paper aims to adopt the GI-OR protocol in greenhouse applications for different sensor types (i.e. temperature, humidity, $\mathrm{CO}_{2}$, Soil). In addition, we compare the results between non-CR WSN, CR WSN and the novel GI-OR CR WSN in terms of energy consumption and network lifetime.

\section{Results and Discussion}

\section{Experimental Setup}

We evaluate the performance of the proposed schemes using Matlab simulator. We consider multiple greenhouses [25 and 30 Greenhouses] in a network and four CRSN types for temperature, humidity, $\mathrm{CO}_{2}$ and soil composed of 6 nodes/type/greenhouse. We assume that the power profile follows that of a typical Atheros Wi-Fi card [7]. Transmission is based on CSMA/CA contention-based access. All channels undergo typical Rayleigh fading Table 1 summarizes the simulation parameters. These parameters are selected based on similar previous works in [12] but with some modifications.

Table 1. Simulation parameters.

\begin{tabular}{ccc}
\hline Definition & \multicolumn{3}{c}{ Value } \\
\hline Number of greenhouses & 25 & \multicolumn{2}{c}{30} \\
Number of CRSN types & \multicolumn{2}{c}{4} \\
Number of nodes/type & 600 & 720 \\
Total number of nodes & \multicolumn{2}{c}{$20 \mathrm{dBm}$} \\
$T_{X}$ power & $-60 \mathrm{dBm}$ \\
Noise Floor & \multicolumn{2}{c}{$-15 \mathrm{dBm}$} \\
Detection Threshold & \multicolumn{2}{c}{$2 \mathrm{slots}$} \\
Sensing Time & \multicolumn{2}{c}{} \\
\hline
\end{tabular}




\section{Energy Consumption}

The energy efficiency in wireless sensors network can be recognized by the ratio of total consumed energy and the number of packet received by the sink node. Here we compare the results between non-CR WSN, CR WSN and the novel GI-OR CR WSN. The WSN energy consumption per node in a single day range from 9 to $18 \mathrm{~mJ}$. The CRSN energy consumption per node in a single day range from 20 to $37 \mathrm{~mJ}$. The GI-OR technique energy consumption per node in a single day range from 5 to $9 \mathrm{~mJ}$. As shown from Figure 3, the GI-OR technique consumes less than half the energy consumed in WSN and a quarter of the energy consumption in CRSN. The energy consumption reduction in GI-OR is due to low packet transmission rate, collisions, and retransmissions.

After a period of time $\mathrm{T}$, some nodes drain their batteries and are treated as dead nodes. In this section, we present the comparison of WSN, CR WSN and CR WSN that uses GI-OR. Figure 4 shows the WSN nodes of 25 Greenhouses after the time period $\mathrm{T}$. Three greenhouses lose observation of data completely.

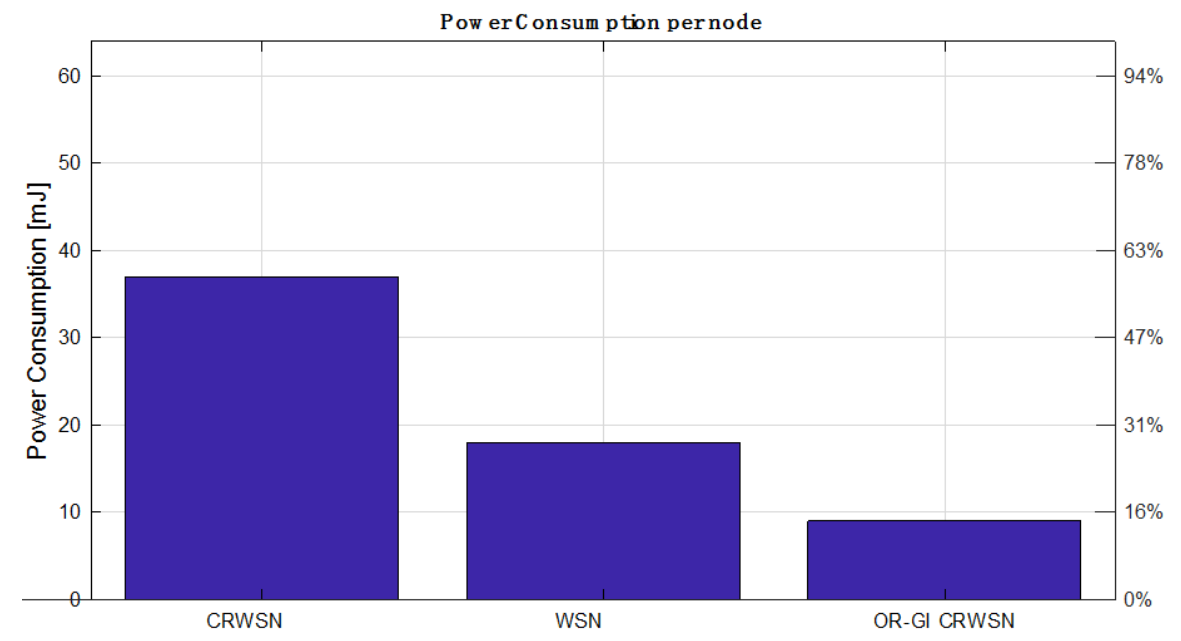

Figure 3. Energy consumption per node.

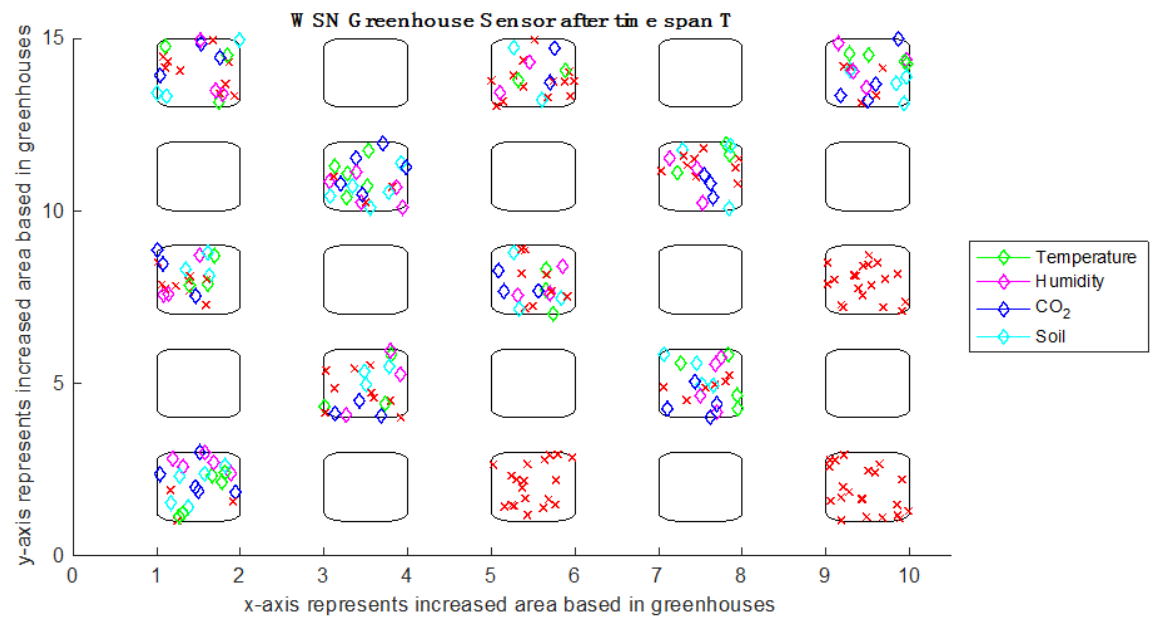

Figure 4. WSN sensors 25 Greenhouses after a period T (Three greenhouses are dead). 
Figure 5 shows the WSN nodes of the 30 Greenhouses after the time period $T$. Four greenhouses lose observation of data completely. Meanwhile, the other greenhouses are still operating in good condition.

Figure 6 and Figure 7 show the CR-WSN nodes of 25 and 30 greenhouses after the time period $T$. In Figure 6, shows many nodes are dead and in Figure 7, too many nodes are dead that due to the CR energy hungry techniques. In contrast, Figure 8 and Figure 9 show the GI-OR protocol nodes of 25 and 30 greenhouses that have low energy consumption and only few of them are dead. Greenhouses, in this case, never lose observation of data in GI-OR CR WSN and all of them are observed.

The network lifetime can be observed for a time period of 36 months for the WSN, CRSN and GI-OR as shown in Figure 10. The existing CRSN techniques drain the nodes battery fast and all network died in 23 months, while the legacy of WSN continued living for 28 months. In contrast, the GI-OR network still lives up to 36 months with about half of the nodes.

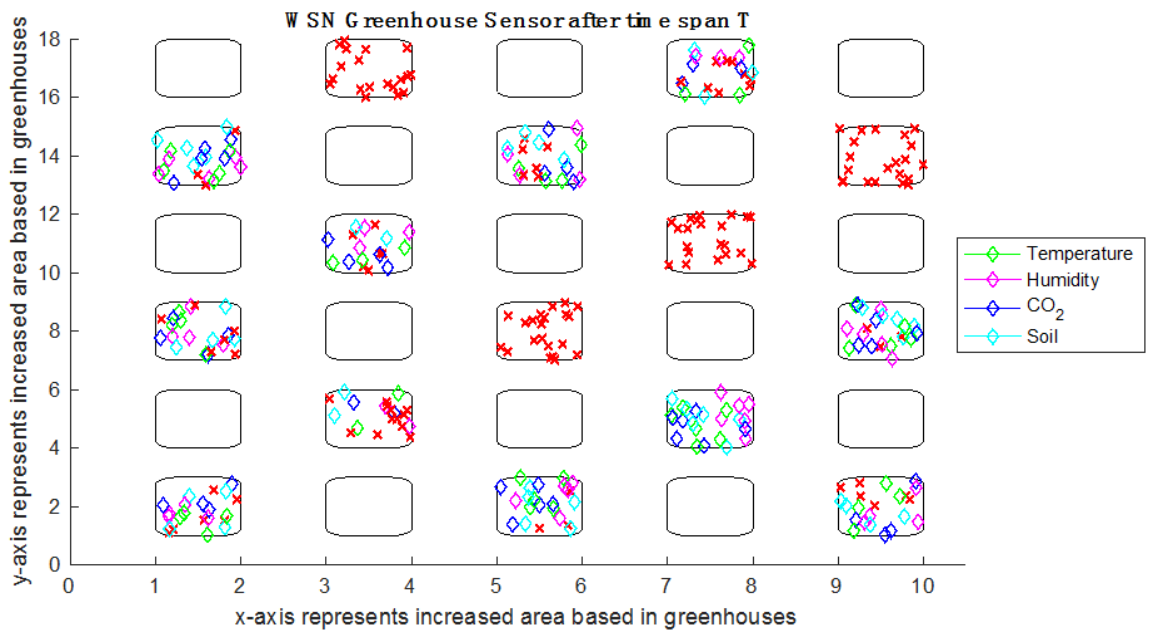

Figure 5. CR WSN sensors 30 Greenhouses after a period T (Four greenhouses are dead).

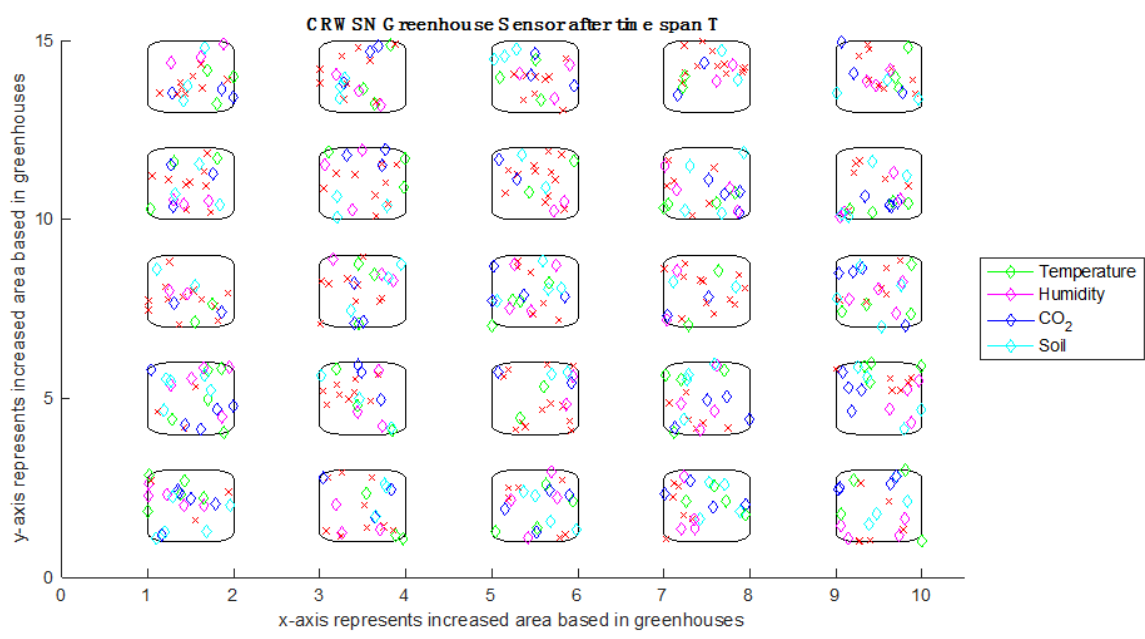

Figure 6. CR WSN sensors of 25 Greenhouses after a period T (many dead nodes). 


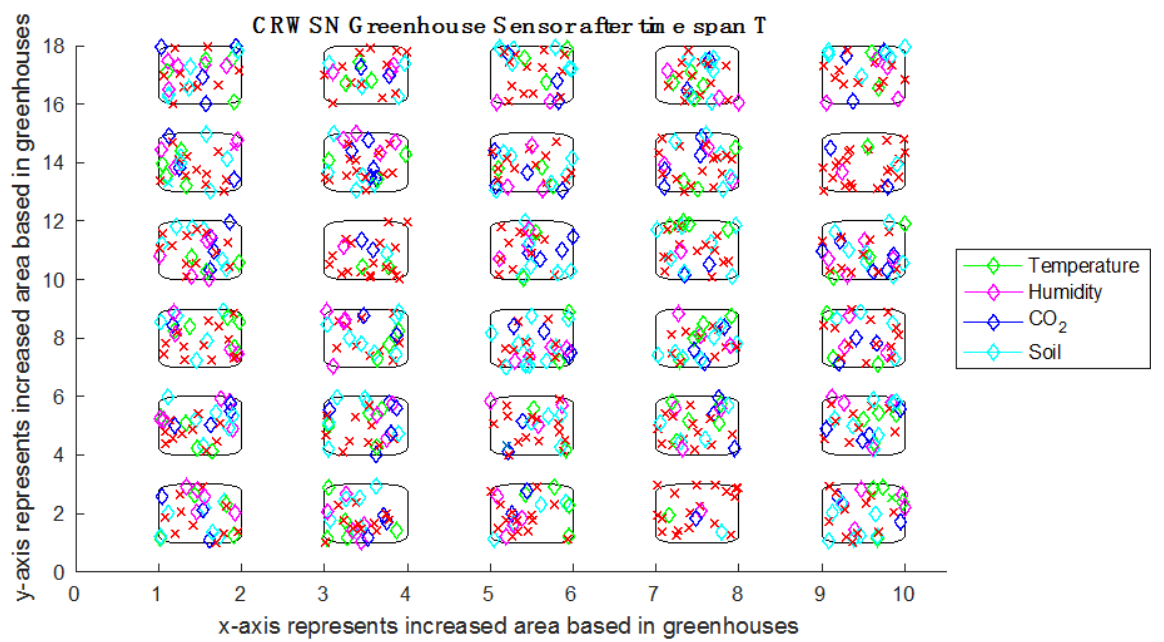

Figure 7. CR WSN sensors of 30 Greenhouses after a period T (too many dead nodes).

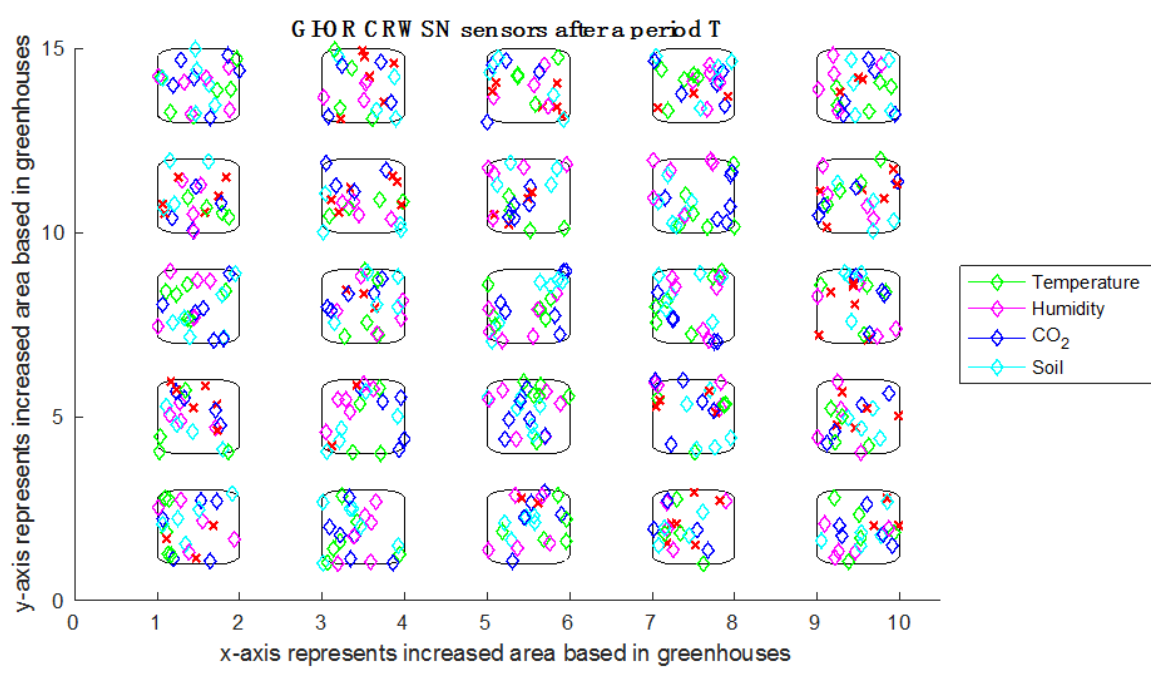

Figure 8. The novel GI-OR CRWSN sensors of 25 Greenhouses after a period T (good and operating).

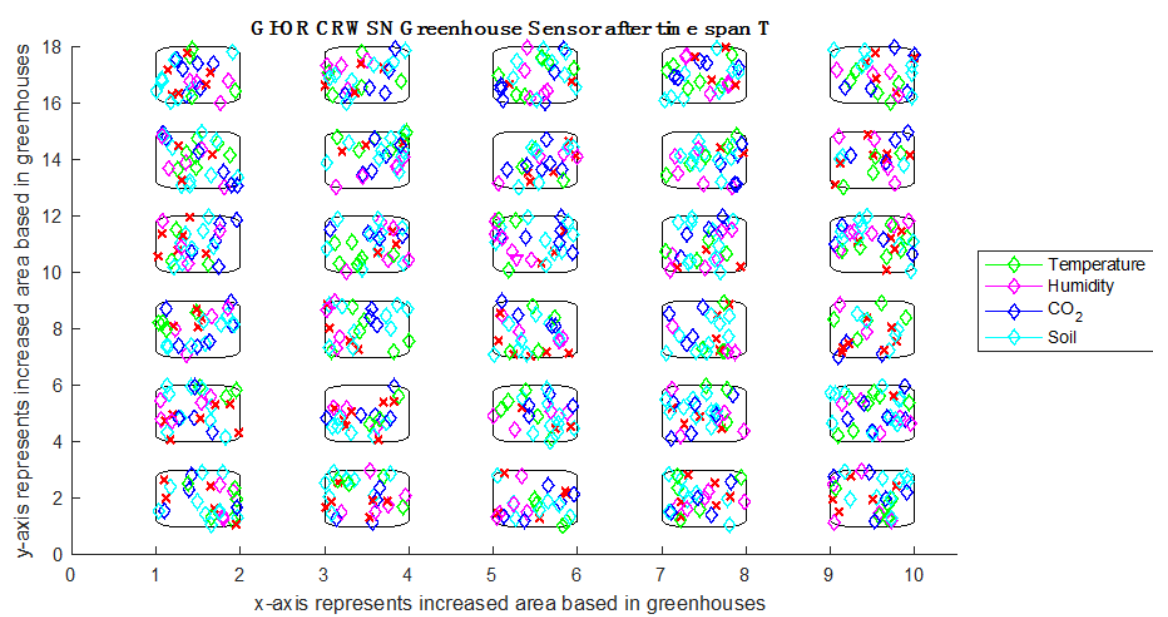

Figure 9. The novel GI-OR CRWSN sensors of 30 greenhouses after a period T (works in perfect condition). 


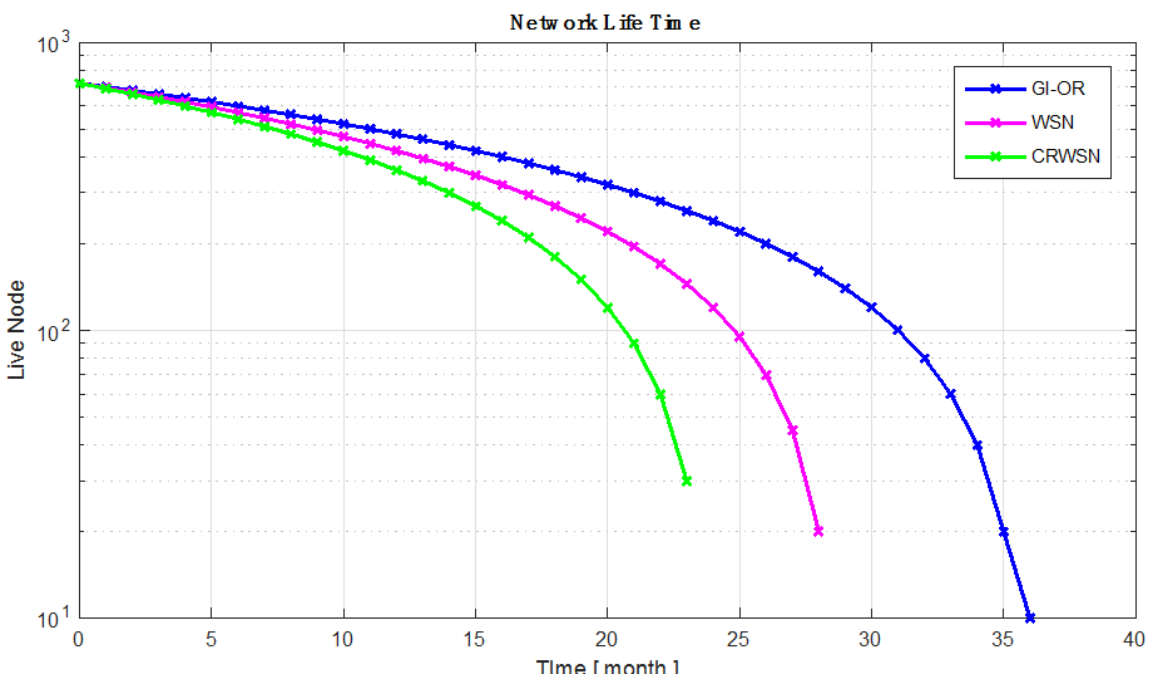

Figure 10. Network lifetime.

\section{Conclusion}

Cognitive radio increased the use of spectrum and the quality of communication with access to opportunistic spectrum, that allowed the user to recognize the best available channel. In this paper, we have presented the efficiency of Generalized Implicit-OR technique in multiple greenhouses. The use of the Generalized Implicit-OR energy protocol enabled the investors to have dense, fully observed greenhouses while the minimum energy consumption and extended the sensor network lifetime was maintained. The proposed Generalized Implicit-OR does not only enhance the consumed energy but also reduced the required capabilities of the nodes to be more simple nodes.

\section{Acknowledgements}

Many thanks to professor Zohdy and all of my committee members, whose served me with my research to be accomplished.

\section{References}

[1] Aytekin, S. and Levent, L. (2016) Greenhouse Automation Using Wireless System. International Journal of Engineering and Computing, 6.

[2] Juang, P., Oki, H., Wang, Y., Martonosi, M., Peh, L.-S. and Rubenstein, D. (2002) Energy-Efficient Computing for Wildlife Tracking: Design Tradeoffs and Early Experiences with ZebraNet. ACM SIGPLAN Notices, 37, 96-107.

[3] Akyildiz, I., Brandon, F. and Balakrishnan, R. (2011) Co-Operative Spectrum Sensing in Cognitive Radio Networks: A Survey. Physical Communications (Elesvier), 4, 40-62. https://doi.org/10.1016/j.phycom.2010.12.003

[4] Cabric, D., Tkachenko, A. and Brodersen, R.W. (2006) Experimental Study of Spectrum Sensing Based on Energy Detection and Network Cooperation. Proceeding TAPAS 06 Proceedings of the First International Workshop on Technology and Policy for Accessing Spectrum, Article No. 12.

[5] Wang, B. and Liu, K.J.R. (2011) Advances in Cognitive Radio Networks: A Survey. 
IEEE Journal of Selected Topics in Signal Processing, 5, 5-23. https://doi.org/10.1109/JSTSP.2010.2093210

[6] Ali, H., Khattab, A. and Fikri, M. (2016) Generalized Implicit Cooperation with Slotted Contention in Cognitive Radio Wireless Sensor Networks. Proceedings of IEEE International Conference on Selected Topics in Mobile \& Wireless Networking (MoWNeT), Cairo, 1-8.

[7] Baradkar, H. and Akojwar, S. (2014) Implementation of Energy Detection Method for Spectrum Sensing in Cognitive Radio Based Embedded Wireless Sensor Net work Node. Proceedings of IEEE ICESC, Nagpur, 9-11 January 2014. https://doi.org/10.1109/ICESC.2014.92

[8] Zhang X. and Shin, K.G. (2012) E-MiLi: Energy-Minimizing Idle Listening in Wireless Networks. IEEE Transactions on Mobile Computing, 11, 1441-1454. https://doi.org/10.1109/TMC.2012.112

[9] Salihu, S.Z.Y.O., et al. (2014) Network Layer for Cognitive Radio Sensor Networks. In: Cognitive Radio Sensor Networks: Applications, Architectures, and Challenges, 196-231.

[10] Zhao, N., et al. (2013) Energy-Efficient Cooperative Spectrum Sensing Schemes for Cognitive Radio Networks. EURASIP Journal on Wireless Communications and Networking, 2013, 1-13. https://doi.org/10.1186/1687-1499-2013-120

[11] Liu, Y., Xie, S., Zhang, Y., Yu, R. and Leung, V.C. (2012) Energy-Efficient Spectrum Discovery for Cognitive Radio Green Networks. Mobile Networks and Applications, 17, 64-74. https://doi.org/10.1007/s11036-011-0307-5

[12] Alhumud, H. and Zohdy, M. (2018) Adopting the Novel Generalized Implicit-OR Sensing Protocol to Decrease the Energy Consumption of Wireless Sensors in Greenhouse. Proceedings of IEEE, International Conference on Electrical, Electronics, Computers, Communication, Mechanical and Computing (EECCMC), Tamil Nadu. 